\title{
Automated ERCC1 immunochemistry on hybrid cytology/tissue microarray of malignant effusions: evaluation of antibodies 8F1 and D-10
}

\author{
Alex Soltermann*, Sandra Kilgus-Hawelski, Silvia Behnke, Martina Storz, Holger Moch and Beata Bode
}

\begin{abstract}
Background: The excision repair cross-complementation group 1 (ERCC1) protein is the key enzyme of the nucleotide excision repair (NER) pathway. Loss of protein expression on immunohistochemistry is predictive for platinum-based chemotherapy response. Frequently, the diagnosis of malignancy is made on cytologic effusion samples. Therefore, we evaluated the staining quality of monoclonal anti-ERCC1 antibodies 8F1 and D-10 on microarrays of malignant pleural and peritoneal effusions by automated immunochemistry.
\end{abstract}

Methods: Cores from effusion cell blocks of 117 patients with > 40 malignant cell clusters per whole section (pleural $n=75$, peritoneal $n=42$ ) were assembled together with 30 histologic control cores from large tissue blocks (lung, breast and ovarian carcinoma, each $n=10$ ) on hybrid cytology-tissue microarrays $(C / T M A)$. Four immunochemistry protocols (Mab 8F1 and D-10, CC1-mono Ventana and H2-60 Bond automat) were performed. Immunoreactivity was semi-quantitatively scored for intensity and intensity multiplied by percentage staining ( $\mathrm{H}$-score).

Results: Tumors were classified into female genital tract carcinoma $(n=39)$, lung adenocarcinoma $(n=23)$, mesothelioma $(n=15)$, unknown primary $(n=14)$, breast carcinoma $(n=10)$, gastro-intestinal carcinoma $(n=12)$ and other $(n=4)$. On both platforms, reproducible nuclear ERCC1 immunoreactivity was achieved with both antibodies, although D-10 was slightly weaker and presented more background staining as well as more variation in the low expression range. No significant differences were found between cytologic and histologic cores. Using the 8F1 CC1-mono protocol, lung and breast carcinomas had lower ERCC1 expression in comparison to the other entities ( $p$-value $<0.05$ ).

Conclusions: Cytology microarrays (CMA) are suitable for investigation of clinical biomarkers and can be combined with conventional TMA's. Dichotomization of ERCC1 immunoreactivity scores is most suitable for patient stratification since definition of negativity is antibody-dependent.

\section{Background}

Platinum-containing drugs like cisplatin are widely used in chemotherapy (CT) regimens of advanced cancers such as ovarian or lung carcinoma due to their robust effectiveness. Cisplatin forms DNA adducts, thereby causing interand intra-strand cross links, comparable to alkylating agents. If not repaired, this DNA damage will lead to apoptotic cell death or mutation. The cross links are removed by trans-lesion synthesis via nucleotide excision repair (NER), which is the primary repair system for bulky

\footnotetext{
* Correspondence: alex.soltermann@usz.ch
Institute of Surgical Pathology, University Hospital Zurich, Schmelzbergstrasse

* Correspondence: alex.soltermann@usz.ch
Institute of Surgical Pathology, University Hospital Zurich, Schmelzbergstrasse 12, CH-8091 Zurich, Switzerland
}

(c) 2011 Soltermann et al; licensee BioMed Central Ltd. This is an Open Access article distributed under the terms of the Creative

DNA lesions caused by such drugs [1]. In the NER system, the heterodimer ERCC1-XPF functions as a structure-specific endonuclease to make the 5 -incision on the damaged strand. This step is claimed to be the key factor [1-3]. Subsequently, a short oligonucleotide fragment containing the offending lesion is replaced. It was deduced that tumors with low nuclear ERCC1 expression better respond to platinum-containing CT because of reduced repair capability for DNA adducts [4,5]. Conversely, patients having tumors with high ERCC1 expression and thus functional NER and also HRR (homologous recombination repair) systems were found to have a better overall survival, since such tumors are assumed to be less unstable and dedifferentiated (so-called ERCC1 paradoxon). Thus, 
ERCC1 is considered an important predictive biomarker for response to platinum-containing CT. A valid predictor of this widely used regimen is of high clinical importance, because response rates in e.g. unselected non-small cell lung cancer (NSCLC) patients range from only 16 to $30 \%$ $[6,7]$.

Assessment of tumoral ERCC1 expression has been performed in different settings, including preclinical, adjuvant and palliative studies $[8,9]$. The results of these studies were controversial. First, differences between mRNA and protein-based studies as well as between formalin-fixed, paraffin-embedded (FFPE) and frozen tissue were observed [10]. Second, protein expression was mostly assessed by immunohistochemistry (IHC) on FFPE tissue, using the mouse monoclonal anti-ERCC1 antibody clone 8F1 [11-14]. However, specificity and intranuclear compartmentalization of this clone was recently challenged $[15,16]$. The ERCC1 predictor concept is now at the point where profound and controlled validation in multi-centre ring-tests be envisaged since this biomarker is used as stratification parameter in oncologic trials. Thus tissue types, tissue processing and protocols of automated immunochemistry platforms need to be standardized.

Importantly, patients having advanced cancers, e.g. originating from ovary, lung or pleura, may primarily present with malignant peritoneal or pleural effusion. Often, the effusion is sent for cytologic diagnosis. Cytologic smears and cell blocks are prepared. No further tissue biopsy may be performed if patients are palliative. Thus, predictors such as EGFR (epidermal growth factor receptor) or ERCC1 are increasingly demanded by clinicians on cytologic material. There are although relevant technical differences between histology and cytology: Histologic sections are 2 to $4 \mu \mathrm{m}$ thick, therefore only a part of the tumor cell nucleus is represented since e.g. NSCLC nuclei have by definition a diameter $>30 \mu \mathrm{m}(>3 \times$ resting lymphocyte diameter) [17]. In contrast, on cytologic smears, entire tumor cells are adherent to the glass slide, thus nuclei are conserved in all 3 dimensions, including $\mathrm{z}$-axis. This fact may lead to major differences when counting nuclear EGFR signals by fluorescence in-situ hybridization (FISH) or semi-quantitatively scoring protein expression intensities by immunohistochemistry. Manufacture of cytologic cell blocks out of the sediment is a means to circumvent cyto-histologic discrepancies since cut thickness is equal.

We have previously investigated the 3 anti-ERCC1 antibodies Mab 8F1, Mab D-10 and Rab FL-297 on a retrospective NSCLC patient cohort assembled on a tissue microarray (TMA) [18]. Only 8F1 and D-10 could be confidently scored. The rabbit polyclonal ab FL-297 presented high cytosolic background and rare weak nuclear staining, thus was omitted. In this study, we aimed for evaluating the staining quality of the $8 \mathrm{~F} 1$ and D-10 antibodies on cytologic effusion cell blocks from most common cancers associated with malignant effusions. Cores from cell blocks and histologic controls were assembled on two hybrid cytology/tissue microarrays (C/TMA) and immunochemistry performed on 2 different automated IHC platforms. We tested the null hypothesis that both antibodies yield similar staining performance due to consistent cut thickness of $4 \mu \mathrm{m}$ across the whole C/TMA surface.

\section{Methods}

\section{Patient cohort}

Cytologic cell blocks of malignant pleural or peritoneal effusions of 125 patients in the time frame 2005-2010, presenting high amounts of malignant cells ( $>40$ clusters per whole section surface) were enrolled in the study. Following diagnostic categories were set up based on morphology, clinical data and immunochemistry with respective markers (using e.g. TTF-1 or Ber-EP4 in case of differential diagnosis between lung adenocarcinoma and mesothelioma): Female genital tract carcinoma (including ovarian, primary peritoneal and uterine carcinoma), lung adenocarcinoma, mesothelioma, breast carcinoma, gastro-intestinal carcinoma (including pancreas, colon and oesophagus carcinoma), unknown primary tumor and other (including squamous cell and large cell carcinoma of the lung, transitional carcinoma of the bladder and rhabdomyosarcoma). On a first C/TMA, 56 tumoral cell block cores were assembled, together with controls $(n=16)$ including benign inflammatory-reactive pleural effusions and histologic tissues from mesothelioma, adenocarcinomas of different organs, transitional cell carcinoma of the bladder and a thoracic lymph node. These controls were not computed. On a second C/ TMA, 69 tumoral cell block cores were assembled together with non-matched control histologic cores from lung, breast and ovarian carcinomas (each $n=10$, total $n$ =30). During processing, malignant cells were lost or immunochemistry was incomplete, respectively, in 8 of 125 cases, thus 117 tumoral cytologic cell block cores from both C/TMAs and all 30 histologic controls from the second could be entirely scored. The study was approved by the institutional review board of the University Hospital Zurich (reference number StV 29-2009).

\section{Cell block}

The effusion liquid was centrifuged at $2000 \times \mathrm{g}$ for 10 min at room temperature and the cell-free supernatant discarded, leaving a small amount of $100 \mu$ liquid above the sediment. The sediment consisted of an upper white phase, containing the tumor cells as well as lymphocytes and mesothelial cells. The lower red phase represented erythrocytes. The upper white phase was aspirated with a Pasteur pipette and few droplets used for manufacture of 3 Papanicolaou stained smears. The rest of the white 
phase was then transferred into a microtube. A clot was quickly formed by addition of 4 droplets plasma (from the hospital's blood donation service) and 1 droplet thrombin (60 NIH-U/ml, Diagnotec AG, Liestal, Switzerland). The clot was transferred into a small inlay cassette with a wooden stick and this cassette was put into a larger histology cassette. After formalin fixation, clots were processed by paraffin embedding and haematoxylin-eosin (H\&E) staining of whole sections.

\section{Hybrid cytology/tissue microarray}

From a representative region of the donor block, a paraffin core of $0.6 \mathrm{~mm}$ diameter and $3-4 \mathrm{~mm}$ height was taken and precisely arrayed into a new recipient paraffin block using a custom-made, semiautomatic tissue arrayer (Beecher Instruments, Sun Prairie, WI, USA). Four $\mu \mathrm{m}$ sections were cut for immunochemistry.

\section{Immunochemistry}

The two mouse monoclonal anti-ERCC1 antibodies 8F1 (Novus Biologicals, Littleton, CO, USA, dilution 1:30) and D-10 (Santa Cruz Biotechnology, Santa Cruz, CA, USA, dilution 1:100), directed against full length protein, were tested on 3 multi-tissue microarrays to select the appropriate dilution as described [18] and further evaluated on whole sections of NSCLC for surface homogeneity. Two automated immunochemistry platforms were used: First, on a Ventana Benchmark ${ }^{\circledR}$ platform (Ventana Medical Systems, Tucson, AZ, USA), the cell conditioner 1 (CC1) standard mono protocol (CC1-mono) was performed: pre-treatment with boiling for $60 \mathrm{~min}$ in $\mathrm{pH} 8$ Tris buffer, incubation with primary ab for $60 \mathrm{~min}$ at room temperature (RT) and development with the Ultraview-HRP mono kit, including incubation with respective secondary ab for $30 \mathrm{~min}$ at RT and additional amplification with respective third and fourth ab. Second, on a Leica Bond ${ }^{\circledR}$ platform (Vision Biosystems, Melbourne, Australia), the H2 standard (H2-60) protocol was performed: pre-treatment with boiling for $60 \mathrm{~min}$ in $\mathrm{pH} 8$ Tris buffer, incubation with primary ab for $30 \mathrm{~min}$ at RT and subsequent development with the Refine-DAB Bond kit, including incubation with respective secondary ab for $30 \mathrm{~min}$ at RT and additional polymer amplification. For TTF-1, the monoclonal antibody SPT24 (Novocastra Laboratories Ltd, Newcastle upon Tyne, UK, dilution 1:100) was used with protocol Ventana CC1-mono. For Ber-EP4 we used Mab M0804 (DakoCytomation, Baar, Switzerland, dilution 1:40) with protocol prediluted protease 1 Ventana and 4 min digestion.

\section{Scoring system}

Nuclear immunoreactivity of both the $8 \mathrm{~F} 1$ and the D-10 ab was scored by A.S. in a blinded manner. The staining intensity was semi-quantitatively scored 0 (negative), 1 (weak), 2 (moderate) or 3 (strong). Further, the percentage of cells having any positivity was proportionally scored 0 (0\%), 0.1 (1-9\%), $0.5(10-49 \%)$ or 1.0 (50\% and more) as described [4]. The H-score was obtained by multiplication of intensity with percentage staining (final range 0 to 3 , per core). Endothelial cells in lymphatic control tissue were assigned an intensity of 2 by default.

\section{Image capture and statistical analysis}

Images were captured on a Zeiss Axioskop connected to a CCD camera, using the image analysis software analySIS FIVE (Olympus BioSystem, Volketswil, Switzerland). White balance was adjusted on analySIS FIVE. No further image processing on Adobe Photoshop such as application of gradation curves for enhancement of contrast or brightness was performed. Correlations of ERCC1 immunoreactivity scores with tumor entities were computed using non-dichotomized data and Kendall's tau-b tests, comparison of score means by the Mann-Whitney U test. P-values $<0.05$ were considered significant. Analyses were carried out on PASW 18.0.0 software package (SPSS Inc., Chicago, IL, USA).

\section{Results}

\section{Cohort description}

Of the 117 patients (pleural effusion $n=75$, peritoneal $n$ = 42) 77 were female and 40 male. The mean age was 66 years (range 29 to 91 years). Table 1 indicates the frequencies of each diagnostic category in both C/TMAs. We concluded that this distribution well represents most common cancers giving rise to malignant effusions and thus is adequate for further investigations.

\section{ERCC1 protein expression on whole sections}

In order to check for surface homogeneity of immunoreactivity, we first stained $4 \mu \mathrm{m}$ thick whole sections of squamous cell lung carcinoma (Figure 1). No image processing such as enhancement of contrast or brightness was performed, except adjustment of white balance. Distinct nuclear staining was achieved with all 4 protocols; however intensity and background varied significantly. Intensity was higher for both antibodies with the H2-60 protocol, although on the cost of increased cytosolic background. The CC1-mono protocol yielded weaker staining, particularly for D-10, but no background. Homogeneous staining was observed over the entire tissue surface. Nuclei were equally stained and no intranuclear compartmentalization was visible apart from omission of nucleoli or nuclear invaginations. Few stroma and necrosis $(<25 \%$ of total surface) was present on the respective whole section, but contributions of immunoreactivity from these compartments were negligible. We concluded that such a 
Table 1 Overview of tissue cores assembled in the $2 \mathrm{C} /$ TMAs, including controls from reactive effusions and histologic solid tumors

\begin{tabular}{lcccc}
\hline & C/TMA $\mathbf{1}$ & C/TMA 2 & \multicolumn{2}{c}{ Total } \\
& $\mathbf{n}$ & $\mathbf{n}$ & $\mathbf{n}$ & $\%$ \\
\hline Tumoral cell blocks & & & & \\
Female Genital Tract Ca & 13 & 26 & 39 & 33.3 \\
Lung Adeno Ca & 12 & 11 & 23 & 19.7 \\
Mesothelioma & 6 & 9 & 15 & 12.8 \\
Unknown Primary & 12 & 2 & 14 & 11.9 \\
Gastro-Intestinal Ca & 3 & 9 & 12 & 10.3 \\
Breast Ca & 3 & 7 & 10 & 8.6 \\
Other & 4 & & 4 & 3.4 \\
Total & 53 & 64 & 117 & 100 \\
\hline Controll
\end{tabular}

Control cell blocks

Reactive pleural effusion

Total 5

Control histology

Female Genital Tract Ca $\quad 2 \quad 10$

Lung Adeno Ca $\quad 1 \quad 10$

Breast Ca

Mesothelioma

Oesophagus Ca

Colon $\mathrm{Ca}$

Lung Squamous Cell Ca

Prostata Ca

Bladder Ca

Thoracic Lymph Node

Total

11

30

surface would be amenable to automated quantitative intensity measurements including creation of a continuous variable.

\section{ERCC1 protein expression on hybrid C/TMA sections}

Consecutive C/TMA sections were first stained for $\mathrm{H} \& \mathrm{E}$ and respective diagnostic markers. Representative images are presented on Figure 2. Second, the four anti-ERCC1 protocols were performed. Both antibodies yielded distinct nuclear signals, although D-10 presented additional focal, but strong background staining of the plasma membrane and intracellular mucin vacuoles (Figure 3). Importantly, many tumor cell clusters were heavily admixed with inflammatory cells also expressing ERCC1 with intensity score 2-3. Inflammatory cells of both malignant and benign effusion sediments stained equally intense compared to lymphatic parenchyma on histologic cores (Figure 4). The same was observed for intratumoral inflammatory infiltrates of histologic solid tumors (data not shown). Further, the cores from the patients with non-malignant control effusions all had reactive mesothelial cells again expressing ERCC1 score 2-3. We concluded that ERCC1 staining intensity of such surfaces is difficult to be quantitatively measured since up to $50 \%$ of immunoreactivity is due to surrounding reactive and inflammatory cells.

\section{Distribution of intensity and $\mathrm{H}$-scores}

For practical reasons, ERCC1 protein expression levels have been dichotomized in most publications closest to the median into low/high, although alternative cut-off's were tested $[4,19]$, since the definition of "ERCC1 negative" is pending. To address this issue, the statistical distributions of the ERCC1 scores were analysed as following: ERCC1 means were consistently found to be in the 1.3 to 2.3 range for all protocols; D-10 antibody incubated with the CC1-mono protocol defining the lower end (Table 2, part A). No significant differences regarding means were found between intensity only and intensity multiplied by percentage of positive cells (H-score). Comparing cytologic versus histologic cores, the average of all 8 means was slightly lower in controls, but this was not significant (pvalue 1.000, Mann-Whitney U test).

Regarding distributions of possible score values in \%, a potential advantage of the $\mathrm{H}$-score was again scarcely visible (Table 2, part B). Consider e.g. the D-10 ab with protocol CC1-mono: Multiplication with percentage of positive cells results in more degrees of freedom, but with regard to dichotomization closest to the median, both intensity and $\mathrm{H}$ scores need to be equally dichotomized 0 to 1 (55.5 and $56.4 \%$ low, respectively) against 2 to 3 (44.5 and $43.6 \%$ high, respectively). Importantly, truly accepting only score 0 and 0.1 as "ERCC1 negative" would mean that for the D-10 antibody from zero (H2-60 protocol, intensity score) to $32.5 \%$ (CC1-mono protocol, $\mathrm{H}$-score) of tumors are negative and thus primarily suited for cisplatin-containing chemotherapy. However, for the 8F1 antibody much less variation was found in the low expression range. Further, using a dichotomization of 0 to 1 versus 2 to 3 , from 43.6\% (D-10 ab, CC1-mono, H-score) to $82.9 \%$ (8F1 ab, $\mathrm{H} 2-60$, intensity score) of tumors would be classified as ERCC1 high. We concluded that statistical distributions of ERCC1 protein expression levels are dependent on technical aspects, in particular selection of antibody and incubation protocol.

\section{Correlation with tumor entities and diagnostic markers}

All ERCC1 scores were next computed against the tumor categories (Table 2, part $\mathrm{C}$ and D). Female genital tract carcinoma and mesothelioma had average score means above 2, whereas the categories breast carcinoma and other marked the lower end. Comparison of cytologic with histologic scores showed similar mean values for breast and female genital tract carcinoma, whereas the lung adenocarcinoma controls had lower expression of ERCC1 compared with cytologic cores. In more detail, we computed scores among lung adeno, breast, female genital tract and gastro-intestinal carcinoma as well as 


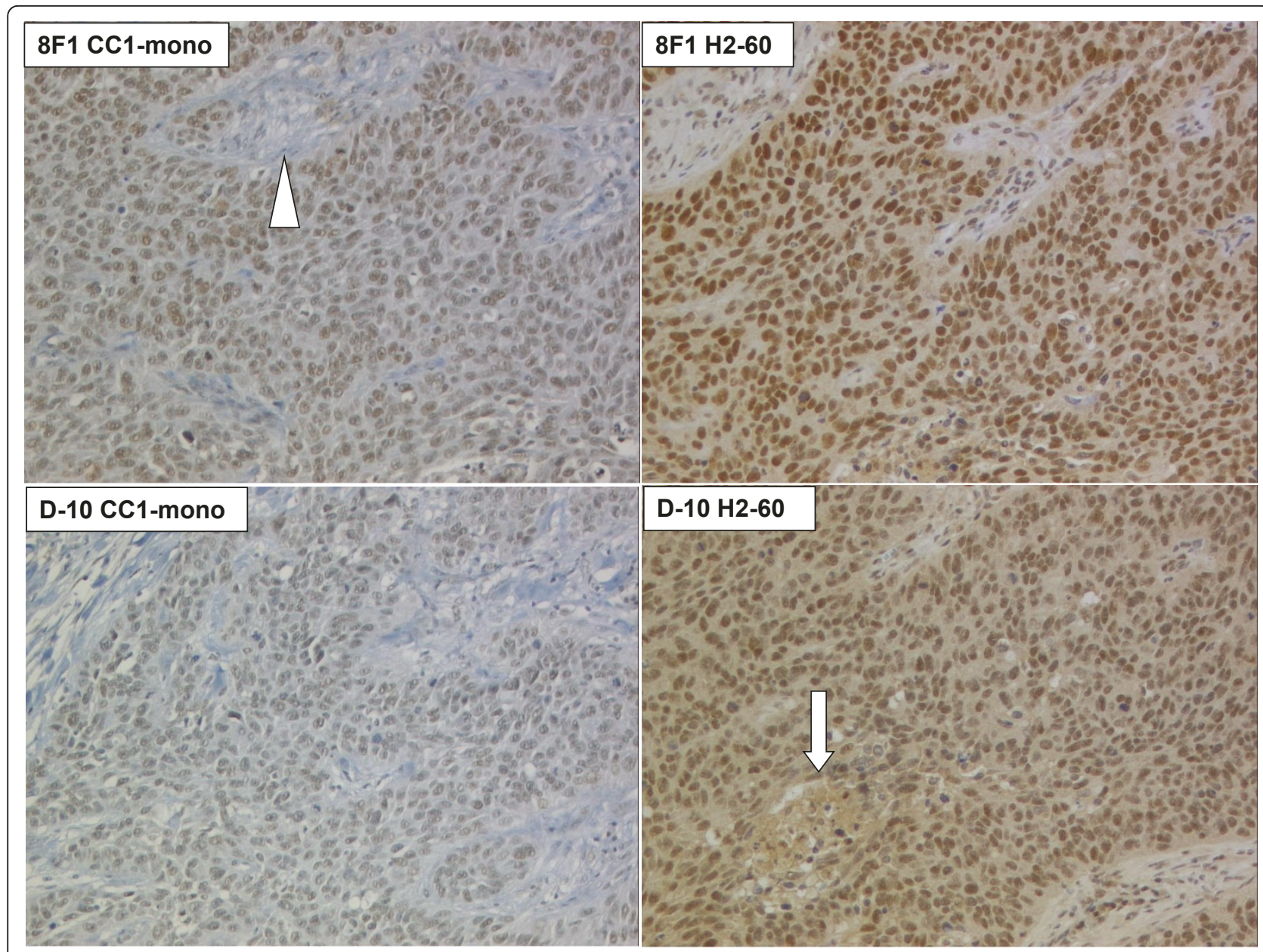

Figure 1 Anti-ERCC1 immunohistochemistry on whole sections of a lung squamous cell carcinoma, using Mab 8F1 and D-10 with CC1mono and H2-60 protocols. Note increased cytosolic background with H2-60. Arrow: Necrotic centre. Arrowhead: Stromal axis. $100 \times$ original magnification.

mesothelioma by Kendall's tau-b tests. The intensity and $\mathrm{H}$-score of the 8F1 antibody with protocol CC1-mono were significantly lower in lung adenocarcinoma and breast carcinoma. However, this relation was not found with any of the other scores. We concluded that cells from most common malignancies giving rise to pleural or peritoneal effusion display a robust ERCC1 protein expression and that no particular entity has completely lost expression of this enzyme.

\section{Discussion}

In this study, we have investigated the immunochemical performance of the 2 mouse monoclonal anti-ERCC1 antibodies $8 \mathrm{~F} 1$ and D-10 on cell blocks of malignant pleural and peritoneal effusions assembled together with histologic control cores to hybrid C/TMAs.

Oncologic trials have started using the ERCC1 expression level as stratification parameter for inclusion into a respective study arm; therefore measurements must be reproducible. Several studies in the preclinical, adjuvant and palliative setting have been performed, using 2 main laboratory approaches: First, patient tumor tissue was examined for ERCC1 expression by either RT-PCR (mRNA) or IHC (protein). Second, tumor tissue or peripheral blood components were genotyped by PCR to examine for SNPs (single nucleotide polymorphism). However, resulting data (comprehensively reviewed in $[8,9])$ is conflicting and entirely opposite correlations were observed. Main reasons for these discrepancies may be differences between fresh frozen and FFPE tissue [10], the small size of bronchial biopsies comprising only few tumor cells and cohort bias due to histotype composition. ERCC1 expression is e.g. higher in squamous cell carcinoma compared to adenocarcinoma. Assessment of SNPs is a new method, mainly investigated in patients with advanced colorectal carcinoma treated with oxaliplatin. Again, e.g. the allelic combination $\mathrm{T} / \mathrm{T}$ was associated on the one hand with a better 


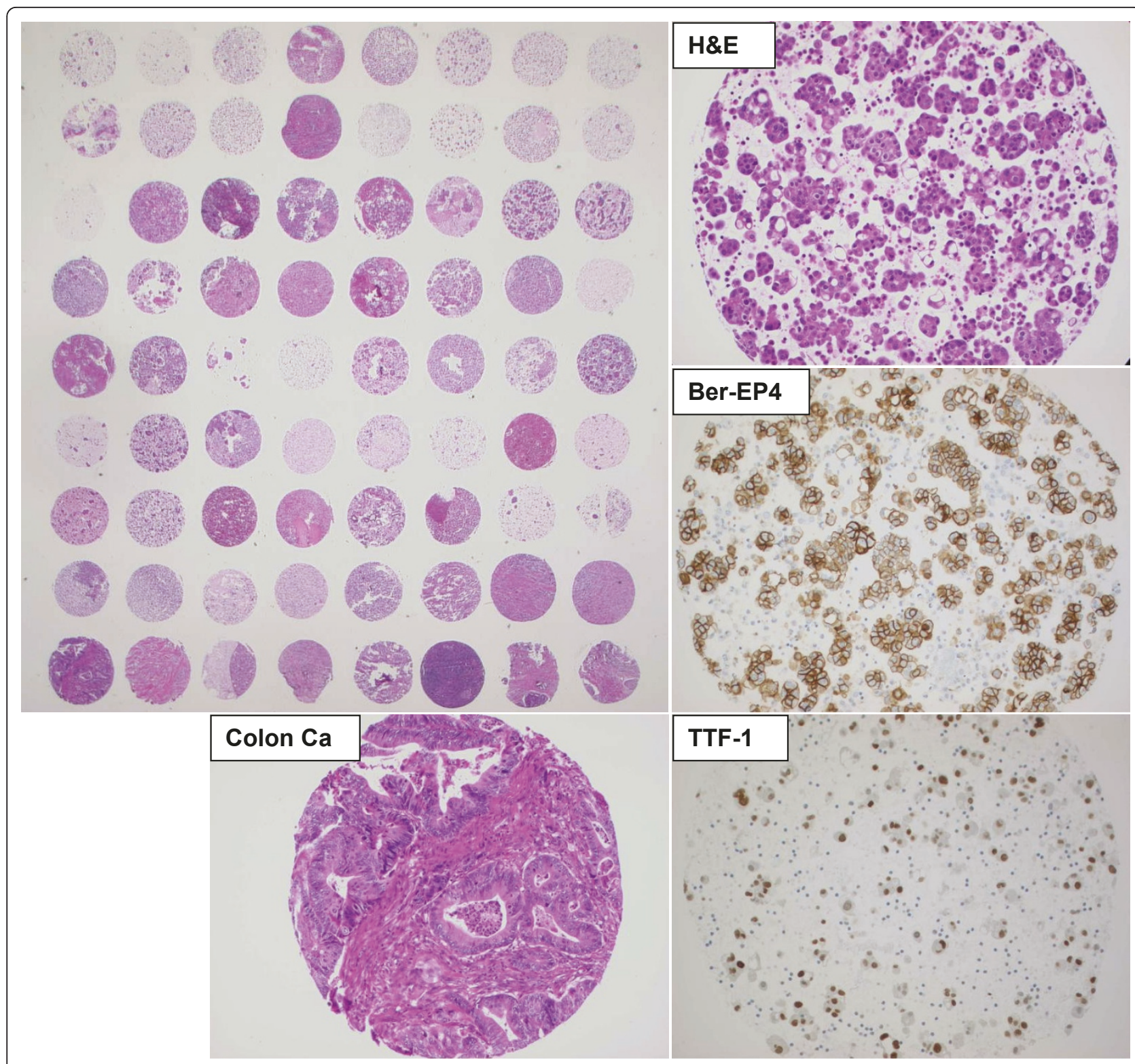

Figure 2 Whole section view of first hybrid C/TMA and representative core from cytologic cell block of a lung adenocarcinoma, stained with H\&E, Ber-EP4 and TTF-1. Lower left: control histologic core of a colon adenocarcinoma. Compare cellular density and thickness of tissue between cytologic and histologic core.

RR (response rate), on the other hand with increased risk of progression [8].

For many patients with advanced cancer, only cytologic smears and corresponding sediments may be available. These sediments can be processed into paraffin cell blocks. The cell block technology has attracted much interest since serial sections, potentially $>100$, can be manufactured and used for assessment of clinically relevant biomarkers, such as EGFR and EML4-ALK (echinoderm microtubule-associated protein-like 4; anaplastic lymphoma kinase) FISH (fluorescent in-situ hybridization) or DNA extraction for PCR of EGFR exons 18-21. Data from these cell block sections is highly comparable to corresponding sections of histologic tissue biopsies or surgical specimens due to the same cut thickness, in most laboratories 2 to $4 \mu \mathrm{m}$. Furthermore, paraffin cores of $0.6 \mathrm{~mm}$ diameter from sediment blocks or also cell line pellets can be assembled into a cytology microarray the same way than cores from histologic blocks into a tissue microarray [20-22]. A cell block may also be an effective quality assurance tool for cancer registries and national mortality statistics [23], since no further diagnostic procedures maybe performed if e.g. a positive pleural effusion defines the pM1a advanced stage of lung adenocarcinoma. 


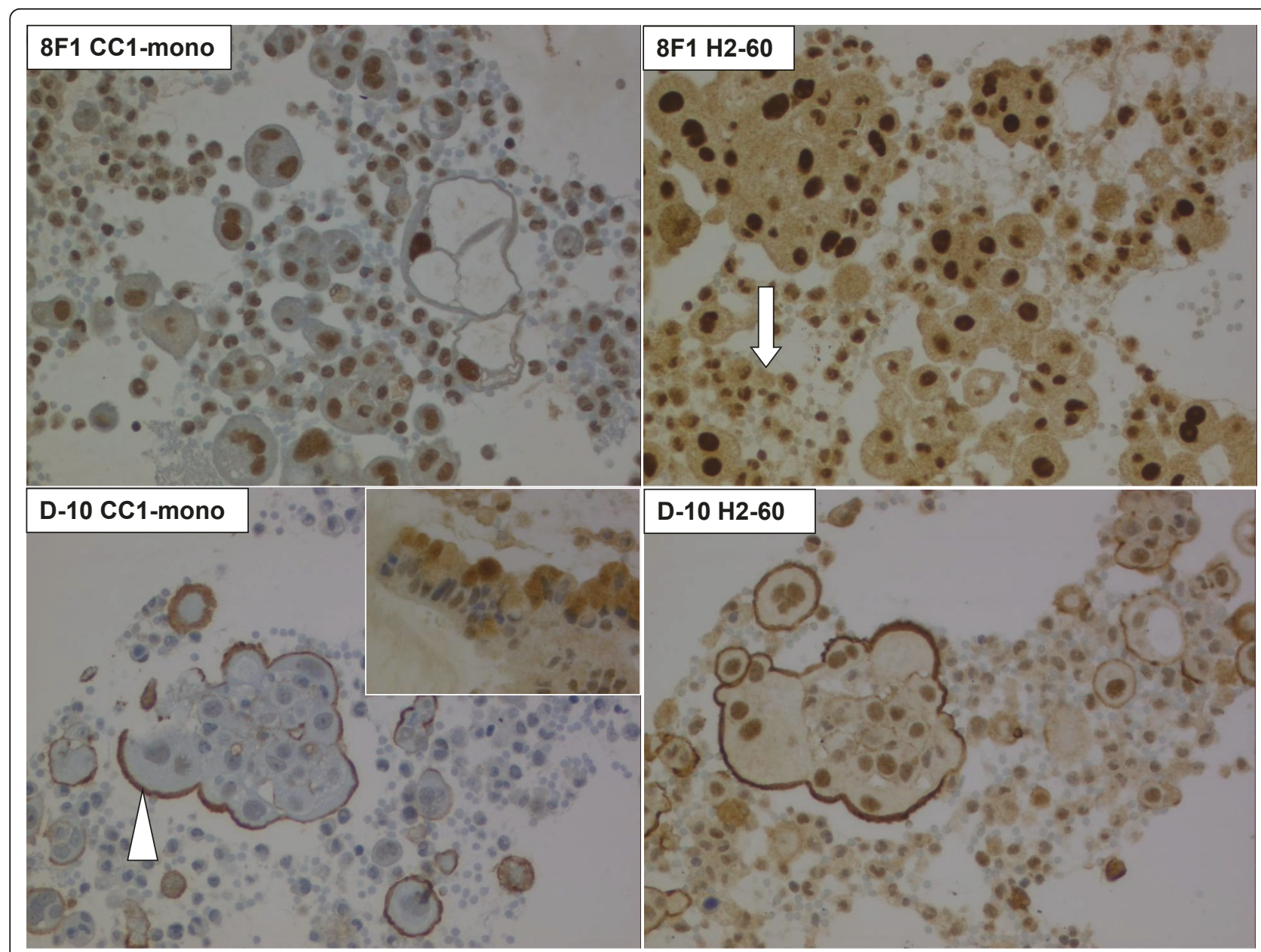

Figure 3 Anti-ERCC1 immunocytochemistry on cell block core of malignant pleural mesothelioma, using Mab 8F1 and D-10 with CC1mono and H2-60 protocols. Arrow: Surrounding non-tumoral cells, including lymphocytes, macrophages and neutrophil granulocytes.

Arrowhead: Unspecific plasma membrane staining with D-10. $200 \times$ original magnification. Inset lower left: Staining of intracellular mucin vacuoles of a mucinous adenocarcinoma of unknown origin. $400 \times$ original magnification.

However, formalin fixation time between cytologic and histologic cores can be significantly different. Clots of tumor cells are quickly formed with plasma/thrombin and often fixed only during the day for some hours. The inlay cassette is then processed the same night on the fixation/staining automat. Conversely, surgical specimens are frequently fixed for up to $48 \mathrm{~h}$ before tissue cuts are loaded on the over-night automat. Thus the major question arises if cytologic cell blocks are usable the same way for biomarker assessment or if additional tissue biopsies need to be taken. Notably, such biopsies are taken only for biomarker investigation and are increasingly considered as integral part of translational research protocols. Ethical concerns have been raised for this strategy and some organs such as lung have not negligible intervention risk. On our hybrid C/TMAs we noticed that inflammatory cells in the effusion sediments had equal staining intensity compared to lymphatic parenchyma or intratumoral inflammatory infiltrate of solid tumors on histologic cores. Further, no significant differences in ERCC1 immunoreactivity were found between tumor cells in effusion liquid and solid sheets on histologic controls. Thus, potential influence of fixation time and depth of fixative penetration seems to be of minor importance.

Currently, immunocytochemistry can be performed on several types of effusion preparations: Ethanol-fixed smears, air-dried and post-fixed cytospins, liquid-based thin layers (ThinPrep), ethanol-fixed cell blocks and formalin-fixed cell blocks. Data on technique superiority is conflicting. Some authors observed best immunoreactivity with ethanol-fixed smears [24]; others experienced equal staining for non-nuclear but superior staining for nuclear markers for formalin-fixed cell blocks in comparison to ThinPrep slides [25]. In general, cell blocks seem to give better morphology and less background staining than cytospins or ThinPrep $[26,27]$ and the use of a 


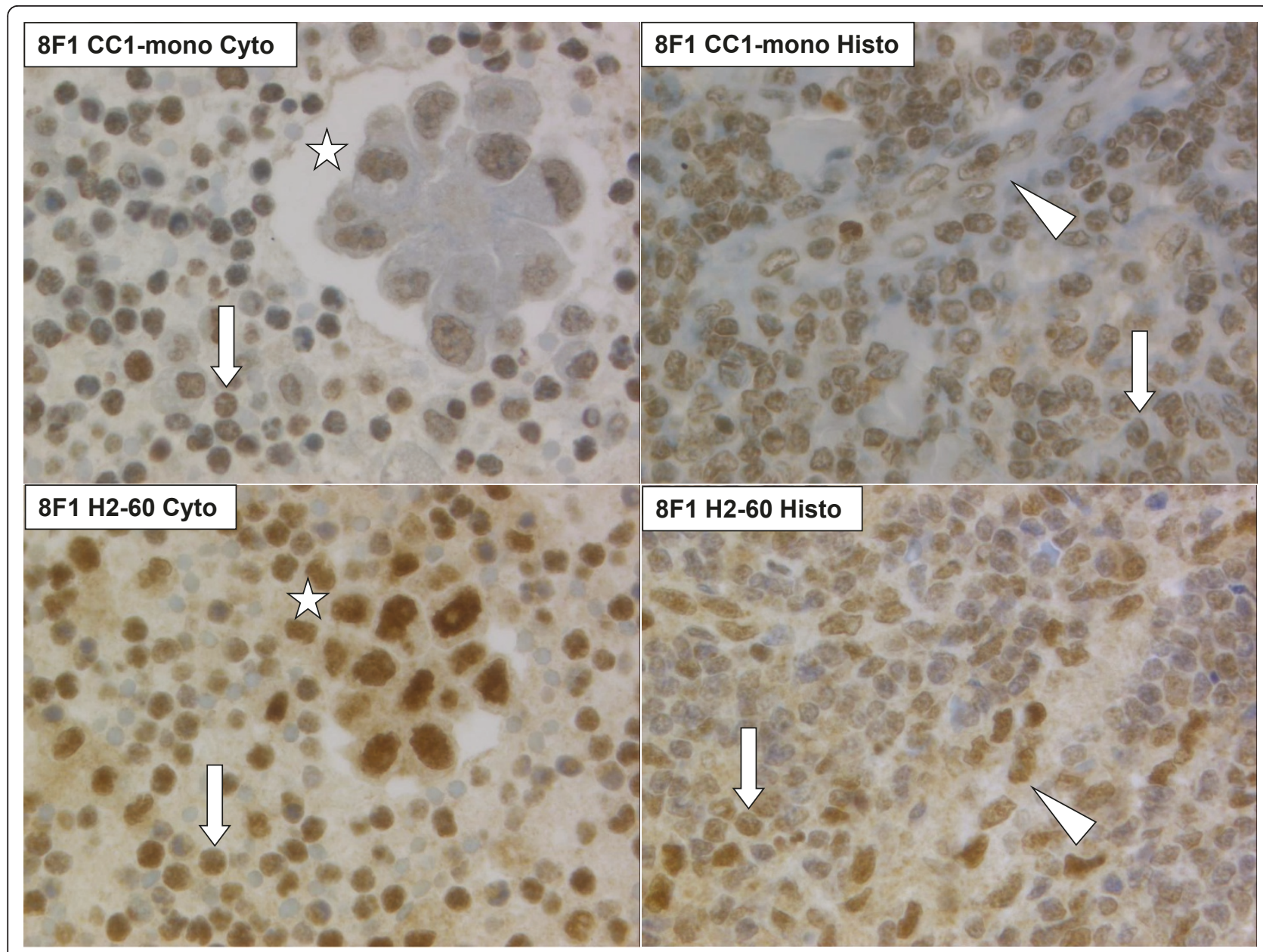

Figure 4 Cyto-histologic comparison of anti-ERCC1 immunoreactivity using the protocol 8F1 CC1-mono (top) or 8F1 H2-60 (bottom). Left: Pleural effusion sediment of lung adenocarcinoma. Right: thoracic lymph node. Arrow: Lymphocyte. Arrowhead: Streak of endothelial cells. Asterisk: Tumor cell cluster. $400 \times$ original magnification.

combined ethanol-formalin fixative has been reported to best preserve the cyto-morphologic features [28]. We thus believe that a formalin-fixation protocol is adequate for a nuclear epitope. Concerning embedding medium, agarose may be used as intermediate [29]. In our protocol a clot is formed by addition of plasma and thrombin to the cells. The question of optimal core diameter and minimal cellularity has been addressed $[29,30]$. The diameter of $0.6,1,2$ or $3 \mathrm{~mm}$ defines the density on the glass slide, but core loss seems to be a minor problem with any diameter. In contrast, cellularity is of major importance when evaluating a larger antibody panel. The distinction into low ( 1 to 20 cell clusters), moderate (20 to 40 cell clusters) and high cellularity ( $>40$ cell clusters), one cell cluster being an aggregate $\geq 5$ cells, seems reasonable and we have implemented the same concept, selecting only blocks with high cellularity. Concerning automated IHC/ICC platforms, the Bond protocol may yield a higher staining intensity due to an in-built polymer amplification step in the detection kit. This is although paid by a slightly increased diffuse background staining. In general, both automated platforms are widely used in routine pathology and reveal sufficient and robust staining for many different antibodies.

Bioinformatics research is ongoing to generate software tools for automated analysis of TMA localization data and XLM-based standardized data capture and transfer [31]. As presented on Figure 2, our hybrid C/TMAs are likely to be amenable to automated localization software. Further, markers such as Ber-EP4 or TTF-1 seem to be suitable for automated quantitative intensity measurements such as AQUA [32-34] or automated image texture analysis [35], due to homogeneous surface staining and absence of co-expressing background inflammatory cells. However, such techniques would be difficult to perform in case of ERCC1 (c.f. Figure 3). Also, parallel protein analysis by immunoblot or mRNA techniques would not alleviate the problem. 
Table 2 Summary of statistical data.

\begin{tabular}{|c|c|c|c|c|c|c|c|c|c|}
\hline & \multicolumn{4}{|c|}{$8 \mathrm{~F} 1$} & \multicolumn{4}{|c|}{ D-10 } & \multirow[b]{3}{*}{ Average } \\
\hline & \multicolumn{2}{|c|}{ CC1-mono } & \multicolumn{2}{|c|}{$\mathrm{H} 2-60$} & \multicolumn{2}{|c|}{ CC1-mono } & \multicolumn{2}{|c|}{$\mathrm{H} 2-60$} & \\
\hline & Int & H & Int & H & Int & H & Int & $\mathrm{H}$ & \\
\hline \multicolumn{10}{|l|}{ A. Score means } \\
\hline Cell blocks & 2.10 & 2.06 & 2.25 & 2.18 & 1.51 & 1.28 & 2.20 & 2.19 & 1.97 \\
\hline Histo controls & 1.63 & 1.57 & 1.97 & 1.92 & 1.63 & 1.46 & 2.23 & 2.08 & 1.81 \\
\hline \multicolumn{10}{|c|}{ B. Distribution \% } \\
\hline \multicolumn{10}{|c|}{ Cell blocks } \\
\hline 0 & 1.7 & 1.7 & 3.4 & 3.4 & 12.8 & 12.8 & 0.0 & 0.9 & 4.6 \\
\hline 0.1 & 0.0 & 0.9 & 0.0 & 4.3 & 0.0 & 19.7 & 0.0 & 0.9 & 3.2 \\
\hline 0.5 & 0.0 & 1.7 & 0.0 & 2.6 & 0.0 & 10.2 & 0.0 & 5.0 & 2.4 \\
\hline 1 & 21.4 & 21.4 & 13.7 & 7.6 & 42.7 & 13.7 & 17.9 & 12.8 & 18.9 \\
\hline 1.5 & 0.0 & 0.0 & 0.0 & 0.0 & 0.0 & 0.0 & 0.0 & 0.9 & 0.1 \\
\hline 2 & 41.9 & 39.3 & 37.6 & 36.8 & 24.8 & 23.9 & 44.5 & 42.7 & 36.3 \\
\hline 3 & 35.0 & 35.0 & 45.3 & 45.3 & 19.7 & 19.7 & 37.6 & 36.8 & 34.3 \\
\hline Total & 100.0 & 100.0 & 100.0 & 100.0 & 100.0 & 100.0 & 100.0 & 100.0 & 100.0 \\
\hline
\end{tabular}

\section{Score means}

\section{Cell blocks}

\begin{tabular}{|c|c|c|c|c|c|c|c|c|c|c|}
\hline & & & & & & & & & & \\
\hline Lung Adeno Ca & & 1.96 & 1.94 & 2.22 & 2.26 & 1.43 & 1.35 & 2.13 & 2.03 & 1.92 \\
\hline Breast Ca & & 1.90 & 1.61 & 1.90 & 1.81 & 1.60 & 1.37 & 1.90 & 1.65 & 1.72 \\
\hline Female Genital Tract Ca & & 2.13 & 2.13 & 2.28 & 2.20 & 1.72 & 1.48 & 2.33 & 2.29 & 2.07 \\
\hline Mesothelioma & & 2.47 & 2.47 & 2.40 & 2.34 & 1.80 & 1.53 & 2.40 & 2.40 & 2.23 \\
\hline Gastro-Intestinal Ca & & 2.33 & 2.33 & 1.92 & 1.83 & 1.25 & 1.10 & 2.00 & 2.00 & 1.85 \\
\hline Unknown Primary & & 1.93 & 1.86 & 2.57 & 2.57 & 1.21 & 0.69 & 2.21 & 2.11 & 1.89 \\
\hline Other & & 1.75 & 1.63 & 2.00 & 2.00 & 0.50 & 0.25 & 1.75 & 1.63 & 1.44 \\
\hline Histologic controls & & & & & & & & & & \\
\hline Lung Adeno Ca & & 1.10 & 0.90 & 1.50 & 1.40 & 1.20 & 0.78 & 1.70 & 1.40 & 1.25 \\
\hline Breast Ca & & 1.90 & 1.90 & 2.00 & 1.95 & 1.60 & 1.50 & 2.50 & 2.35 & 1.96 \\
\hline Female Genital Tract Ca & & 1.90 & 1.90 & 2.40 & 2.40 & 2.10 & 2.10 & 2.50 & 2.50 & 2.23 \\
\hline D. Correlat.ion entities & & & & & & & & & & \\
\hline Lung AdenoCa & $\mathrm{p}$ & 0.019 & 0.009 & ns & ns & ns & ns & ns & ns & ERCC1 lower \\
\hline Breast Ca & tau & 0.186 & 0.201 & & & & & & & Lung Adeno \\
\hline Female Genital Tract Ca & & & & & & & & & & and Breast $\mathrm{Ca}$ \\
\hline Mesothelioma & & & & & & & & & & \\
\hline Gastro-Intestinal Ca & & & & & & & & & & \\
\hline
\end{tabular}

A. Means of intensity and H-scores across cytologic $(n=117)$ and histologic $(n=30)$ cores. B. Distributions (\%) of score values. C. Means of scores among tumor entities on both cytologic and histologic cores. D. Correlation of ERCC1 scores with tumor entities (Kendall's tau-b test used).

In the original paper of ERCC1 IHC on human FFPE tissue of NSCLC patients, the Mab 8F1 was used [4]. The specificity of this antibody was although recently challenged $[15,16]$, since $8 \mathrm{~F} 1$ stained a second spurious band on immunoblots from human fibroblasts but not HeLa cervical carcinoma cells and could not discriminate between ERCC1-positive and negative fibroblasts on immunofluorescence. However, 8F1 confidently detected His-tagged purified ERCC1. In reply, the authors of the first NSCLC study demonstrated that in the HeLa and the A549 lung adenocarcinoma cell lines, one major band of $36 \mathrm{kD}$ was observed on immunoblot using 8F1 and this band disappeared after siRNAmediated depletion [11]. In this study, both $8 \mathrm{~F} 1$ and D-
10 homogeneously and robustly stained the whole nuclear surface. No intranuclear compartmentalization was observed apart from omission of nucleoli or nuclear invaginations. However, D-10 showed unspecific background staining at the plasma membrane and in intracellular mucin vacuoles and was generally weaker on same protocols.

\section{Conclusions}

In summary, cell block cytology microarrays (CMA) are suitable for investigations of relevant clinical biomarkers and can be mixed with TMA's to yield C/TMA hybrids. On the two automated IHC/ICC platforms Ventana Benchmark $^{\circledR}$ and Leica Bond ${ }^{\circledR}$, the anti-ERCC1 antibody 
8F1 performed superior compared to D-10 in terms of staining quality and restriction to the nuclear compartment.

\section{Acknowledgements}

We would like to thank P. Cione for excellent technical assistance in manufacturing cell blocks.

\section{Authors' contributions}

AS carried out the immunochemical scoring, performed statistical analysis and drafted the manuscript together with $\mathrm{HM}$. SKH and BB diagnosed patients and assembled the cohort. SB carried out the immunochemistry, MS manufactured the C/TMAs. All authors read and approved the final manuscript.

\section{Competing interests}

The authors declare that they have no competing interests.

Received: 6 September 2010 Accepted: 30 September 2011 Published: 30 September 2011

\section{References}

1. de Laat WL, Jaspers NG, Hoeijmakers JH: Molecular mechanism of nucleotide excision repair. Genes Dev 1999, 13:768-785.

2. Friedberg EC: How nucleotide excision repair protects against cancer. Nat Rev Cancer 2001, 1:22-33

3. Martin LP, Hamilton TC, Schilder RJ: Platinum resistance: the role of DNA repair pathways. Clin Cancer Res 2008, 14:1291-1295.

4. Olaussen KA, Dunant A, Fouret P, Brambilla E, Andre F, Haddad V, et al: DNA repair by ERCC1 in non-small-cell lung cancer and cisplatin-based adjuvant chemotherapy. N Engl J Med 2006, 355:983-91.

5. Soria JC: ERCC1-tailored chemotherapy in lung cancer: the first prospective randomized trial. J Clin Oncol 2007, 25:2648-2649.

6. Schiller JH, Harrington D, Belani CP, Langer C, Sandler A, Krook J, et al: Comparison of four chemotherapy regimens for advanced non-small-cell lung cancer. N Engl J Med 2002, 346:92-98.

7. Fossella F, Pereira JR, von Pawel J, Pluzanska A, Gorbounova V, Kaukel E, et al: Randomized, multinational, phase III study of docetaxel plus platinum combinations versus vinorelbine plus cisplatin for advanced non-small-cell lung cancer: the TAX 326 study group. J Clin Oncol 2003, 21:3016-3024.

8. Vilmar A, Sørensen JB: Excision repair cross-complementation group 1 (ERCC1) in platinum-based treatment of non-small cell lung cancer with special emphasis on carboplatin: A review of current literature. Lung Cancer 2009, 64:131-139.

9. Gossage L, Madhusudan S: Current status of excision repair cross complementing-group 1 (ERCC1) in cancer. Cancer Treat Rev 2007, 33:565-577.

10. Booton R, Ward T, Ashcroft L, Morris J, Heighway J, Thatcher N: ERCC1 mRNA expression is not associated with response and survival after platinum-based chemotherapy regimens in advanced non-small cell lung cancer. J Thorac Oncol 2007, 2:902-906.

11. Olaussen KA, Fouret P, Kroemer G: ERCC1-specific immunostaining in nonsmall-cell lung cancer. N Engl J Med 2007, 357:1559-1561.

12. Zheng Z, Chen T, Li X, Haura E, Sharma A, Bepler G: DNA synthesis and repair genes RRM1 and ERCC1 in lung cancer. N Engl J Med 2007, 356:800-808.

13. Lee HW, Han JH, Kim JH, Lee MH, Jeong SH, Kang SY, et al: Expression of excision repair cross-complementation group 1 protein predicts poor outcome in patients with small cell lung cancer. Lung Cancer 2007, 59:95-104.

14. Kwon HC, Roh MS, Oh SY, Kim SH, Kim MC, Kim JS, et al: Prognostic value of expression of ERCC1, thymidylate synthase, and glutathione Stransferase P1 for 5-fluorouracil/oxaliplatin chemotherapy in advanced gastric cancer. Ann Oncol 2007, 18:504-509.

15. Niedernhofer $L$, Bhagwat N, Wood RD: ERCC1 and non-small-cell lung cancer. N Engl J Med 2007, 356:2538-2540.
16. Bhagwat NR, Roginskaya WY, Acquafondata MB, Dhir R, Wood RD, Niedernhofer LJ: Immunodetection of DNA repair endonuclease ERCC1XPF in human tissue. Cancer Res 2009, 69:6831-6838.

17. Wiatrowska BA, Krol J, Zakowski MF: Large-cell neuroendocrine carcinoma of the lung: proposed criteria for cytologic diagnosis. Diagn Cytopathol 2001, 24:58-64.

18. Arbogast S, Behnke S, Opitz I, Stahel R, Seifert B, Weder W, et al: Automated ERCC1 immunohistochemistry in non-small cell lung cancer: Comparison of anti-ERCC1 antibodies 8F1, D-10 and FL-297. Appl Immunohistochem Mol Morphol 2010, Oct. 27, E-pub.

19. Holm B, Mellemgaard A, Skov T, Skov BG: Different impact of excision repair cross-complementation group 1 on survival in male and female patients with inoperable non-small-cell lung cancer treated with carboplatin and gemcitabine. J Clin Oncol 2009, 27:4254-4259.

20. Bubendorf L: Tissue microarrays meet cytopathology. Acta Cytol 2006, 50:121-122.

21. Pu RT, Giordano TJ, Michael CW: Utility of cytology microarray constructed from effusion cell blocks for immunomarker validation. Cancer 2008, 114:300-306.

22. Wirth GJ, Schandelmaier K, Smith V, Burger AM, Fiebig HH: Microarrays of 41 human tumor cell lines for the characterization of new molecular targets: expression patterns of cathepsin B and the transferrin receptor. Oncology 2006, 71:86-94.

23. Hsu FD, Nielsen TO, Alkushi A, Dupuis B, Huntsman D, Liu CL, et al: Tissue microarrays are an effective quality assurance tool for diagnostic immunohistochemistry. Mod Pathol 2002, 15:1374-1380.

24. Ueda J, Iwata T, Ono M, Takahashi M: Comparison of three cytologic preparation methods and immunocytochemistries to distinguish adenocarcinoma cells from reactive mesothelial cells in serous effusion. Diagn Cytopathol 2006, 34:6-10.

25. Gong Y, Sun X, Michael CW, Attal S, Williamson BA, Bedrossian CW: Immunocytochemistry of serous effusion specimens: a comparison of ThinPrep vs cell block. Diagn Cytopathol 2003, 28:1-5.

26. Fetsch PA, Simsir A, Brosky K, Abati A: Comparison of three commonly used cytologic preparations in effusion immunocytochemistry. Diagn Cytopathol 2002, 26:61-66.

27. Pereira TC, Saad RS, Liu Y, Silverman JF: The diagnosis of malignancy in effusion cytology: a pattern recognition approach. Adv Anat Pathol 2006, 13:174-184.

28. Nathan NA, Narayan E, Smith MM, Horn MJ: Cell block cytology. Improved preparation and its efficacy in diagnostic cytology. Am J Clin Pathol 2000, 114:599-606.

29. Wen CH, Su YC, Wang SL, Yang SF, Chai CY: Application of the microarray technique to cell blocks. Acta Cytol 2007, 51:42-46.

30. Anagnostou VK, Lowery FJ, Syrigos KN, Cagle PT, Rimm DL: Quantitative evaluation of protein expression as a function of tissue microarray core diameter: is a large $(1.5 \mathrm{~mm})$ core better than a small $(0.6 \mathrm{~mm})$ core? Arch Pathol Lab Med 2010, 134:613-619.

31. Dhir R: Tissue microarrays: an overview. Methods Mol Biol 2008, 441:91-103.

32. Gustavson MD, Bourke-Martin B, Reilly D, Cregger M, Williams C, Mayotte J, et al: Standardization of HER2 immunohistochemistry in breast cancer by automated quantitative analysis. Arch Pathol Lab Med 2009, 133:1413-1419.

33. Moeder CB, Giltnane JM, Moulis SP, Rimm DL: Quantitative, fluorescencebased in-situ assessment of protein expression. Methods Mol Biol 2009, 520:163-175

34. Rojo MG, Bueno G, Slodkowska J: Review of imaging solutions for integrated quantitative immunohistochemistry in the Pathology daily practice. Folia Histochem Cytobiol 2009, 47:349-354.

35. Karacali B, Tozeren A: Automated detection of regions of interest for tissue microarray experiments: an image texture analysis. BMC Med Imaging 2007, 7:2.

doi:10.1186/2043-9113-1-25

Cite this article as: Soltermann et al: Automated ERCC1

immunochemistry on hybrid cytology/tissue microarray of malignant effusions: evaluation of antibodies 8F1 and D-10. Journal of Clinical

Bioinformatics 2011 1:25. 